ARTICLE

\title{
The effect of water on colloidal quantum dot solar cells
}

Guozheng Shi ${ }^{1}$, Haibin Wang (10 2 , Yaohong Zhang ${ }^{3}$, Chen Cheng (1) ${ }^{1}$, Tianshu Zhai ${ }^{1}$, Botong Chen ${ }^{1}$, Xinyi Liu ${ }^{4}$, Ryota Jono (1) 2, Xinnan Mao', Yang Liu' ${ }^{1}$, Xuliang Zhang ${ }^{1}$, Xufeng Ling (1) ${ }^{1}$, Yannan Zhang ${ }^{1}$, Xing Meng ${ }^{1}$, Yifan Chen', Steffen Duhm (1) 1, Liang Zhang (1) 1, Tao Li (i) 4,5, Lu Wang (1) 1, Shiyun Xiong1, Takashi Sagawa (1) 6 , Takaya Kubo², Hiroshi Segawa², Qing Shen ${ }^{3}$, Zeke Liu (i] ${ }^{1 凶} \&$ Wanli Ma (i] ${ }^{1 凶}$

Almost all surfaces sensitive to the ambient environment are covered by water, whereas the impacts of water on surface-dominated colloidal quantum dot (CQD) semiconductor electronics have rarely been explored. Here, strongly hydrogen-bonded water on hydroxylated lead sulfide ( $\mathrm{PbS}$ ) CQD is identified. The water could pilot the thermally induced evolution of surface chemical environment, which significantly influences the nanostructures, carrier dynamics, and trap behaviors in CQD solar cells. The aggravation of surface hydroxylation and water adsorption triggers epitaxial CQD fusion during device fabrication under humid ambient, giving rise to the inter-band traps and deficiency in solar cells. To address this problem, meniscus-guided-coating technique is introduced to achieve dense-packed CQD solids and extrude ambient water, improving device performance and thermal stability. Our works not only elucidate the water involved PbS CQD surface chemistry, but may also achieve a comprehensive understanding of the impact of ambient water on CQD based electronics.

\footnotetext{
${ }^{1}$ Institute of Functional Nano and Soft Materials (FUNSOM), Jiangsu Key Laboratory for Carbon-Based Functional Materials and Devices, Joint International Research Laboratory of Carbon-Based Functional Materials and Devices, Soochow University, Suzhou, Jiangsu, China. ${ }^{2}$ Research Center for Advanced Science and Technology, The University of Tokyo, Meguro-ku, Tokyo, Japan. ${ }^{3}$ Faculty of Informatics and Engineering, The University of ElectroCommunications, Tokyo, Japan. ${ }^{4}$ Department of Chemistry and Biochemistry, Northern Illinois University, DeKalb, IL, USA. ${ }^{5}$ X-ray Science Division, Argonne National Laboratory, Lemont, IL, USA. ${ }^{6}$ Graduate School of Energy Science, Kyoto University, Kyoto, Japan. ${ }^{凶}$ email: zkliu@suda.edu.cn; wlma@suda.edu.cn
} 
T he presence of water on solid surfaces is ubiquitous in nature, which significantly impacts the surface chemical process and the corresponding properties of metals, oxides, and semiconductors through surface hydroxylation and water adsorption ${ }^{1-3}$. The surface-dominant nature of colloidal quantum dots (CQDs) endows them extreme surface sensitivity towards ambient humidity ${ }^{4}$. It has been reported that humid ambient has significant impacts on the properties of CQDs ${ }^{5-8}$. Uncovering the effect of water should be essential to open the door towards the ambient manufacturing of CQD-based electronic devices. However, the water-involved surface chemistry and its potential influence on CQD electronics have rarely been explored yet.

Semiconducting lead sulfide (PbS) CQDs are promising building blocks for solution-processed electronics, including photovoltaics, infrared photodetectors, and field-effect transistors ${ }^{9}, 10$. The surface geometry plays a critical role in the CQD surface chemistry and performance of devices, which can be predicted by Wulff constructions based on surface energy minimization theorem ${ }^{11,12}$. The surface of PbS CQD with a small size of less than $3 \mathrm{~nm}$ is dominated by polar $\{111\}$ facet. Further growth of CQDs will lead to the appearance of $\{100\}$ and $\{110\}$ facets. This feature of CQD with controllable surface geometries provides a model platform for the study of the water effect on CQD characteristics, starting from the atom-level surface chemistry to the macroscopic photovoltaic properties.

Generally, the environmental water molecules can hydroxylate the solid surface via dissociative chemisorption during material synthesis or ambient device fabrication ${ }^{13,14}$. In 2014, Zherebetskyy et al. demonstrated that the dissociated water molecules could cause surface hydroxylation during PbS CQDs synthetic process ${ }^{15}$. The interposition of the hydroxyl anions $(\mathrm{OH})$ between steric oleic acid (OA) ligands effectively releases the surface energy of $\{111\}$ facet, preserving facet stabilization. The strong bonding between surface $\mathrm{Pb}$ and the hydroxyls on $\{111\}$ facet make $\mathrm{OH}$ hard to be removed by the conventional ligand exchange process ${ }^{16}$. The discovery of partially hydroxylated surfaces has deepened the understanding of the surface science in $\mathrm{PbS}$ CQDs. Thereafter, close associations between the surface $\mathrm{OH}$ and the deficiency in solar cell performance have been observed. Cao et al. achieved improvement in power conversion efficiency (PCE) and photostability of solar cells based on iodine-passivated $\mathrm{PbS}$ (PbS-I) CQD by effectively reducing the surface $\mathrm{OH}$ ligands ${ }^{17}$. Followed by this innovation, strategies were developed to avoid the adverse effect of surface hydroxyls, including precursor engineering ${ }^{16}$, direct synthesis of CQD inks ${ }^{18}$, protic solvent control ${ }^{19}$, ink engineering ${ }^{20}$, and the introduction of etching agent ${ }^{21-23}$. Although the investigation results suggest that the surface $\mathrm{OH}$ may lead to trap states, the identification of involved hydroxyl species and their exact connections with the defectiveness in PbS CQDs are still substantial subjects of debate ${ }^{11,24,25}$. So far, there is no theoretical or experimental evidence to support that the surface $\mathrm{OH}$ ligands could directly serve as the trap sources. Thus, it remains an urgent challenge to understand the pathways from ambient water to $\mathrm{PbS}$ surface hydroxylates and eventually to the reduced device performance.

Subsequently, the hydroxylation on solid surfaces allows the adsorption of ambient water by hydrogen bonding between the surface $\mathrm{OH}$ and the water molecules, forming a configuration of mixed $\mathrm{OH}+\left(\mathrm{H}_{2} \mathrm{O}\right)_{n}{ }^{26-28}$. The adsorbed water on solid surfaces with a thickness ranging from a few $\AA$ to infinite bulk may participate in surface chemical reactions ${ }^{29}$. However, the water adsorption on hydroxylated PbS CQDs under humid air has been ignored so far. Thus, the investigation of adsorbed water may shed new light on the surface chemistry and the mystery of hydroxyl-induced device deficiency in PbS CQD solar cells.
In this study, $\mathrm{H}$-bonded water is identified for the first time on the partially hydroxylated surface of PbS CQDs by in-situ temperature-dependent X-ray photoelectron spectroscopy (XPS), Xray absorption spectroscopy (XAS), and the density functional theory (DFT) studies. The results indicate that the adsorbed water could govern the temperature-dependent change of CQD surface chemistry through water desorption, hydroxylation, and dehydration process. More importantly, we reveal that exposure to ambient water during device processing will enhance the surface hydroxylation and water adsorption, which subsequently aid the fusion of adjacent CQDs. The inter-band trap states and energetic disorder introduced by fused CQDs will harm the performance of CQD solar cells, which unambiguously elucidate the correlation between surface $\mathrm{OH}$ ligands and device deficiency. We also reveal that the notorious thermal instability of PbS CQD solar cells may originate from the continuous fusion, as well as surface iodine loss and migration during thermal aging. To resolve the issue, the meniscus-guided deposition technique, i.e., convective assembly (CA), was used instead of spin-coating to achieve morphological homogeneity of CQD films. Resultantly, the densely packed CQD arrays could spatially extrude the ambient water and thus reduce surface hydroxylates, leading to improved device performance and thermal stability. We believe our works not only elucidate hydroxyl involved CQD surface chemistry but also achieve a comprehensive understanding of the impact of ambient water on CQD solar cells, which may provide general insight into the practical manufacturing of stable CQD based electronics via a scalable manner under ambient air.

\section{Results}

Identification of surface hydroxylates. Figure la shows the geometry structure of truncated octahedron PbS CQD used in our study. The surfaces of $\sim 3 \mathrm{~nm}$ PbS CQD used in our studies are mainly dominated by $\mathrm{PbS}\{111\}$ facets, with partial coverage of $\{100\}$ facets. In ideal situations, the CQD should be fully covered by iodine on the polar $\{111\}$ facet. However, the strong steric hindrance of $\mathrm{OA}$ allows partial surface hydroxylation, stabilizing $\mathrm{PbS}\{111\}$ facets during the synthesis process ${ }^{15}$. The surface $\mathrm{OH}$ could provide potential absorption sites for ambient water, which makes CQD more vulnerable to the ambient environment.

To theoretically predict the water adsorption on CQD surfaces, we employ the slab models including $\mathrm{PbS}\{111\},\{100\}$, and $\{110\}$ facets covered by different surface species as the geometric configurations in our density functional theory (DFT) calculations, as illustrated in Fig. 1b and Supplementary Fig. $1^{11}$. By calculating the adsorption energy $\left(E_{\mathrm{ad}}\right)$ between water molecules and CQD surfaces, the most stable adsorbate configuration can be revealed ${ }^{30}$. The $E_{\text {ad }}$, density of states (DOS), and bonding motifs of $\{111\}$ surfaces are displayed and summarized in Supplementary Fig. 2 and Supplementary Table 1. For the fully iodine-passivated $\mathrm{PbS}$ facet, a negligible $E_{\mathrm{ad}}$ suggests no capability for water absorption. As for the partially hydroxylated facets, preferable water adsorption on surface $\mathrm{OH}$ ligands can be predicted by a low $E_{\text {ad }}$ of $-0.51 \mathrm{eV}$ per $\mathrm{H}_{2} \mathrm{O}$. A clean band edge without mid-gap states introduced by surface $\mathrm{OH}$ or H-bonding is suggested by DOS in Supplementary Fig. 3, which indicates that $\mathrm{OH}$ or adsorbed water molecules will not directly introduce surface trap states. It is worth noting that the oxidized facet exhibits the strongest capability of water adsorption (Supplementary Fig. 2c). The large electronegativity of oxygen atom may pull the hydrogen in water molecule to the neighbor $\mathrm{Pb}-\mathrm{O}$ site, forming two hydroxyls through a reverse $\mathrm{OH}$ recombination reaction ${ }^{31}$.

To experimentally identify the surface hydroxylates, systematical X-ray spectroscopy studies were performed. The in-situ temperaturedependent XPS measurement provides experimentally traceable clues 


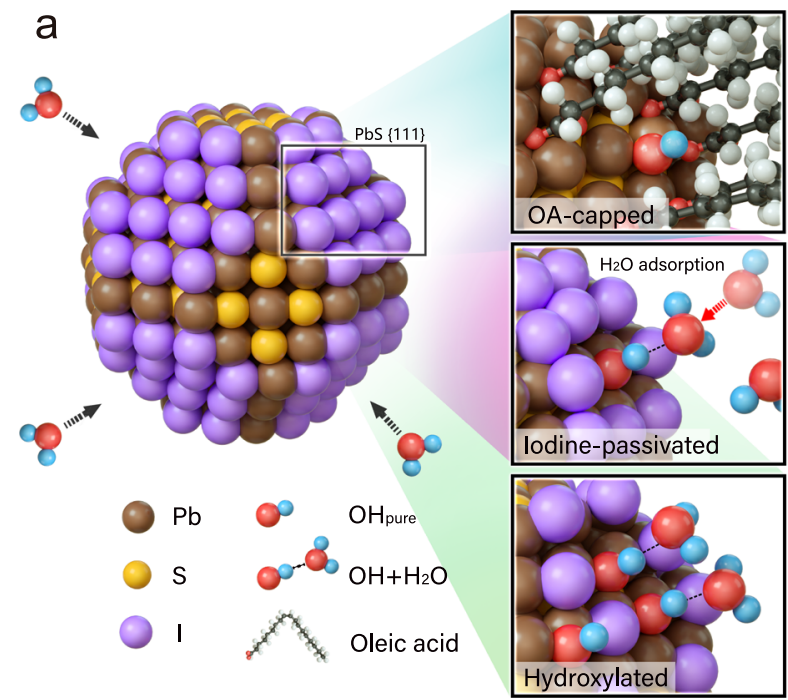

b
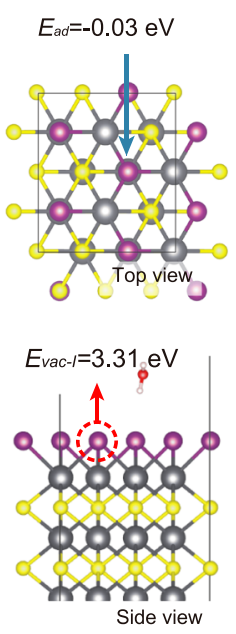

lodine passivated
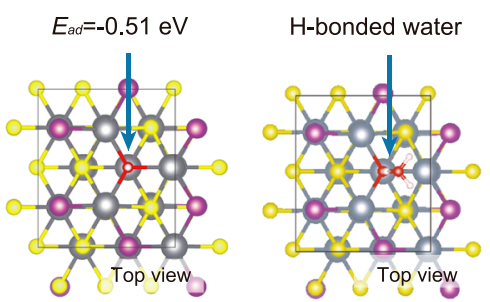

$E_{\text {vac }-I}=3.37 \mathrm{eV}$
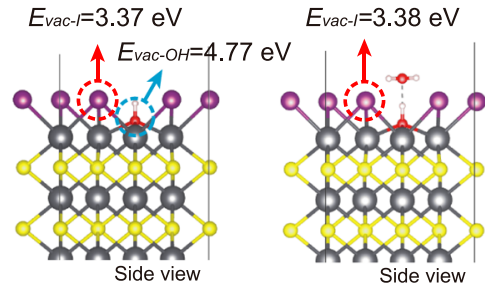

Partially hydroxylated
Water adsorbed
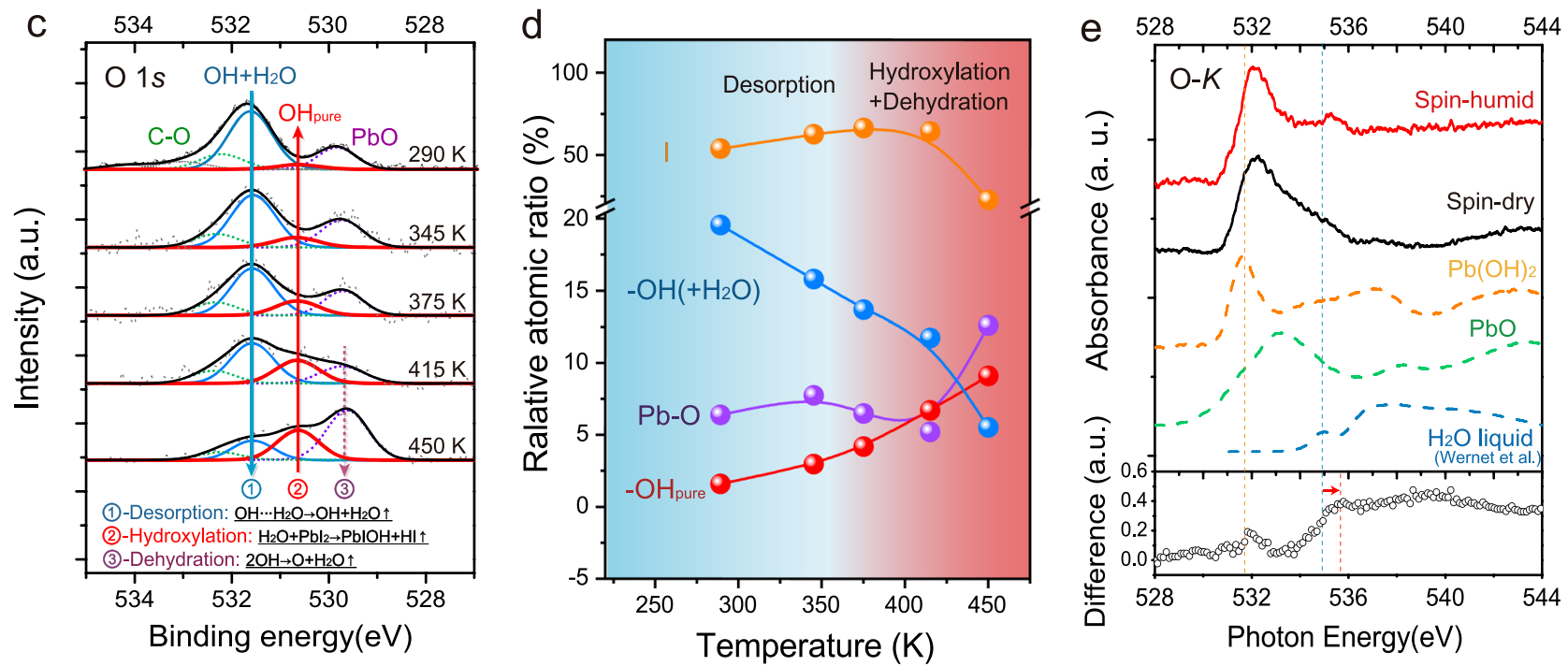

Fig. 1 Identification of water adsorption on partially hydroxylated CQD surfaces. a Schematic representation of surface conditions on octahedral PbS CQDs. The ideal model of PbS CQDs with atomic halide passivation is shown on the left. The proposed surface conditions on the Pb-terminated $\{111\}$ facet are zoomed on the right. Top: the OA-capped facet with partial surface hydroxylation introduced in the synthetic process ${ }^{15}$; Medium: Atomic iodinepassivated facet after ligand exchange; Below: the aggravation of surface hydroxylation followed by water adsorption under humid air. $\mathbf{b}$ The geometric structures of iodine-passivated (left), partially hydroxylated (middle) and water adsorbed PbS $\{111\}$ facets (right) used in DFT calculation. The $E_{\text {ad }}$ and $E_{\mathrm{vac}}$ stand for the adsorption energy of $\mathrm{H}_{2} \mathrm{O}$ and the vacancy formation energy, respectively. The purple spheres stand for iodine atoms, black ones for Pb atoms, yellow ones for $\mathrm{S}$ atoms, red ones for $\mathrm{O}$ atoms, and white ones for $\mathrm{H}$ atoms. c The temperature-dependent O1s XPS spectra of PbS-I film. $\mathbf{d}$ Atomic ratio of surface species relative to $\mathrm{Pb} 4 f$ core recorded at a temperature from $290 \mathrm{~K}$ to $450 \mathrm{~K}$. e XAS spectra of PbS CQD films at the oxygen K-edge prepared under different ambient conditions. The "Spin-humid" and "Spin-dry" represent the sample deposited and ligand exchanged under ambient air with a high $\mathrm{RH}(50-60 \%)$ or low $\mathrm{RH}(<10 \%)$, respectively. The XAS spectra of liquid water and different surface chemical species observed in XPS O1s core are also shown. The XAS spectra of liquid water are extracted from Wernet et al. (2004) ${ }^{2}$. Reprinted with permission from AAAS. The spectra difference between "Spin-humid" and "Spin-dry" is calculated for a clearer comparison.

for the water chemistry on the CQD surface, where the heating under ultra-high vacuum (UHV) could kinetically overcome the activation barrier for a specific chemical reaction ${ }^{32}$. Figure $1 \mathrm{c}$ illustrates the temperature-dependent O $1 s$ spectra of iodine-passivated PbS CQD film prepared by solid-state-ligand-exchanged (SSLE) method measured at $290-450 \mathrm{~K}$. The sample was spin-coated under ambient condition (40-50\% relative humidity). The center peak with the binding energy $(\mathrm{BE})$ at $531.6 \mathrm{eV}$ (acquired at $290 \mathrm{~K}$ ) is generally assigned to oxygen in $\mathrm{OH}$-groups, the peak centered at $529.8 \mathrm{eV}$ to $\mathrm{Pb}-\mathrm{O}$, and the high $\mathrm{BE}$ shoulder to $\mathrm{C}-\mathrm{O}$ species $^{33}$. However, with increasing temperature, a new peak at $530.7 \mathrm{eV}$ emerges and the $\mathrm{OH}$ peak at $531.6 \mathrm{eV}$ decreases. The unexpected peak evolution suggests that the $\mathrm{OH}$-related surface species on PbS CQDs are more complex than originally assumed in literatures, as summarized in Supplementary Table $3^{15}$. Similar spectra change have been observed in the in-situ temperature-dependent XPS studies of water adsorption on metal surfaces ${ }^{32}$. We thus reassign the original $\mathrm{OH}$ peak at $531.6 \mathrm{eV}$ to the mixed $\mathrm{OH}+\mathrm{H}_{2} \mathrm{O}$ phases formed by hydrogen bonding between the surface $\mathrm{OH}$ and water molecules, as supported by our DFT results. Consequently, we ascribe the new peak emerging at $530.7 \mathrm{eV}$ with increasing temperature to the pure hydroxyl groups on $\mathrm{PbS}$ CQD surfaces due to water desorption, noted as $\mathrm{OH}_{\text {pure }}$ 
For the partially hydroxylated PbS CQD surface with iodide passivation, we reveal that water plays a critical role in the thermally induced evolution of surface chemistry under UHV by three steps: (1) The desorption of surface absorbed water; (2) the aggravation of surface hydroxylation; (3) the dehydration of surface hydroxyls. Figure 1d shows the atomic ratio of surface species relative to $\mathrm{Pb} 4 f$ with increasing temperatures from $290 \mathrm{~K}$ to $450 \mathrm{~K}$. The corresponding XPS spectra and atom ratio are summarized in Supplementary Fig. 4 and Supplementary Table 4. Briefly, a trade-off between $\mathrm{OH}_{\text {pure }}$ and $\mathrm{OH}+\mathrm{H}_{2} \mathrm{O}$ species on the CQD surface is observed, which could proceed through a desorption process of $\mathrm{H}$-bonded water by:

$$
\mathrm{OH} \cdots \mathrm{H}_{2} \mathrm{O} \stackrel{\Delta}{\longrightarrow} \mathrm{OH}_{\text {pure }}+\mathrm{H}_{2} \mathrm{O} \uparrow \text { (Desorption) }
$$

Upon transition of surface hydroxylates from mixed $\mathrm{OH}+$ $\mathrm{H}_{2} \mathrm{O}$ phases to $\mathrm{OH}_{\text {pure }}$ at high temperature, the surface iodide decomposes quickly, as reflected by the sharply reduced $\mathrm{I} / \mathrm{Pb}$ ratio at high temperature (Supplementary Table 4$)^{34-36}$. The serious iodine loss may partially stem from the thermally induced Schottky vacancies. It is also likely that the remaining water will participate in a basic salt reaction at a high temperature above $415 \mathrm{~K}$ :

$$
\mathrm{H}_{2} \mathrm{O}+\mathrm{PbI}_{2} \stackrel{\Delta}{\longrightarrow} \mathrm{PbIOH}+\mathrm{HI} \uparrow \text { (Hydroxylation) }
$$

In this case, the adsorbed water on the CQD surface may not merely work as a "spectator", namely, being extracted into the vacuum after desorption, but rather work as the reactants in hydroxylation process, significantly replacing the iodine by $\mathrm{OH}_{\text {pure }}$. Similar chemical evolution has been identified in the water-associated decomposition process of lead halide perovskite, forming a subsalt of lead hydroxide iodide $(\mathrm{PbIOH})$ under humid ambient ${ }^{37}$. Meanwhile, the $\mathrm{OH}_{\text {pure }}$ ligands could form the $\mathrm{Pb}-\mathrm{O}$ species via an $\mathrm{OH}$ recombination reaction at elevated temperatures, with the resultant atomic $\mathrm{O}$ coordinated with neighbor $\mathrm{Pb}$ on facets: $:^{38}$

$$
\mathrm{OH}_{\text {pure }}+\mathrm{OH}_{\text {pure }} \stackrel{\Delta}{\longrightarrow} \mathrm{O}+\mathrm{H}_{2} \mathrm{O} \uparrow \text { (Dehydration) }
$$

The $\mathrm{Pb}-\mathrm{O}$ accumulation and I loss are consistent with the results in Fig. 1d. The in-situ temperature-dependent XPS unveils the CQD surface chemistry under UHV conditions, which indicates the critical role of adsorbed water in the evolution of surface species.

We found that the surface hydroxylation not only happened during the synthesis process but could also occur during device fabrication under humid air. The ex-situ XPS measurements of CQD films with or without annealing under dry or humid ambient are conducted, as shown in Supplementary Fig. 5 and Supplementary Table 5. With the aid of gentile annealing, drastic growth of surface hydroxylates under the humid atmosphere is obtained. A spontaneous reaction can be involved:

$$
2 \mathrm{H}_{2} \mathrm{O}+\mathrm{PbI}_{2}+\frac{1}{2} \mathrm{O}_{2} \rightarrow 2 \mathrm{~Pb}(\mathrm{OH})_{2}+\mathrm{I}_{2} \uparrow \text { (Hydroxylation under humid air) }
$$

This reaction on CQD $\mathrm{PbS}\{111\}$ facet is theoretically supported by the reduction of total potential energy from DFT calculations $(\Delta E=-94.40 \mathrm{~kJ} / \mathrm{mol})$, as shown in Supplementary Fig. 6a, suggesting reaction (4) is energetically preferable on CQD surfaces. The resultant surface hydroxyls can be stabilized by the following $\mathrm{H}$-bonding formation with adsorbed water, further reducing the system energy by $-48.96 \mathrm{~kJ} / \mathrm{mol}$. The theoretical prediction agrees well with the XPS experimental results, as shown in Supplementary Fig. 6c-d. The continuous heating of PbS-I CQD film leads to nearly full-surface hydroxylation and I loss. A similar chemical routine is also suggested as one of the decay pathways of lead halide perovskites under humid ambient ${ }^{39}$.

To confirm the water adsorption and further understand the water bonding motifs on hydroxylated $\mathrm{PbS} C \mathrm{CQD}$ surface, $\mathrm{O} \mathrm{K}$ edge X-ray spectra (XAS) are measured and shown in Fig. 1e. The XAS has been extensively used to detect the water configuration due to its high sensitivity to the $\mathrm{H}$-bond distortions on the $\mathrm{H}$ sides of water molecules $2,40,41$. The $\mathrm{O}$ K-edge XAS spectra of $\mathrm{Pb}$ $(\mathrm{OH})_{2}, \mathrm{PbO}$, and liquid $\mathrm{H}_{2} \mathrm{O}$ are presented as references ${ }^{2}$. A dominant pre-edge feature is observed between $531 \mathrm{eV}$ to $532 \mathrm{eV}$ in the XAS spectrum of the spin-coated PbS-I samples prepared under dry air, which is mainly contributed by $\mathrm{Pb}-\mathrm{O}$ and $\mathrm{Pb}-\mathrm{OH}$ species. For the sample prepared under humid air (RH 50-60\%), a new peak around $535.3 \mathrm{eV}$ emerges, attributed to the adsorbed surface water. The distinction of surface species between spin-dry and spin-humid samples can be further identified in the difference spectrum (lower row of Fig. 1e). The sharp peak at $531.9 \mathrm{eV}$ can be ascribed to the difference in $\mathrm{Pb}-\mathrm{OH}$ surface species varied by ambient humidity. The notable peak near 535.5 $\mathrm{eV}$ is caused by water adsorption, while it shows an energy shift of $0.7 \mathrm{eV}$ relative to the peak near the pre-edge of liquid water (blue dashed line, Fig. 1e). Generally, the liquid water molecules exhibit local coordination with only one strongly $\mathrm{H}$-bonded $\mathrm{OH}$ group, reflected by a distinct pre-edge feature in XAS, whereas ice shows fully coordinated hydroxyls characterized by XAS spectra with only post-edge peak ${ }^{2}$. Thus, the peak shift in the difference spectra indicates that a certain amount of adsorbed water molecules may form two hydrogen bonding with the surrounding $\mathrm{OH}$, transforming from a single donor (liquid) to double donor (ice) configuration on the CQD surface ${ }^{2,40}$.

Resultantly, a schematic surface illustration of truncated octahedral PbS CQD is shown in Fig. 1a. The hydroxylates on $\mathrm{PbS}$ CQD surfaces can be formed under ambient conditions via three routes: (1) the strong steric hindrance of $\mathrm{OA}$ allows partial surface hydroxylation to stabilize the $\mathrm{PbS}\{111\}$ facet during the synthesis process (Supplementary Fig. 7, formula S$1)^{15}$. The hydroxyls are hard to be removed during the ligand exchange process due to the strong $\mathrm{Pb}-\mathrm{OH}$ bonding ${ }^{16}$; $(2)$ the exposure of hydroxylated surfaces to the ambient water, leading to water adsorption by H-bonding (formula S-2, S-7); (3) The hydroxylation on iodine-passivated CQD facets during ambient processing accelerated by gentile annealing, along with the subsequent surface adsorption of ambient water (formula S6, S-7).

Water impacted morphology in CQD solids. The ambient water can not only guide the surface chemistry process but also change the morphology in CQD solids at different spatial scales. Figure 2a-e demonstrates the high-resolution transmission electron microscope (TEM) images of $\mathrm{PbS}$ CQDs prepared under different conditions. Under dry air, the substitutions of long OA ligand by atomic iodine passivants shorten the inter-dot spacing but keep CQDs intact after heating for $30 \mathrm{~min}$ at $85^{\circ} \mathrm{C}$ (Fig. 2b). In comparison, under humid air, apparent epitaxial CQD fusion along with increasing heating time is observed in Fig. 2c-e. In this case, hydroxylation and subsequent water adsorption on CQD surfaces can be aggravated, as presented in Supplementary Fig. 5d. Serious CQD fusion was also found in the heavily hydroxylated $\mathrm{PbS}$ CQDs treated by tetramethylammonium hydroxide pentahydrate $\left(\mathrm{TMAOH} \cdot 5 \mathrm{H}_{2} \mathrm{O}\right)$ methanol solution ${ }^{42}$ even without further annealing process (Supplementary Fig. 8c), suggesting the close link between surface hydroxylates and CQD fusion. 
a

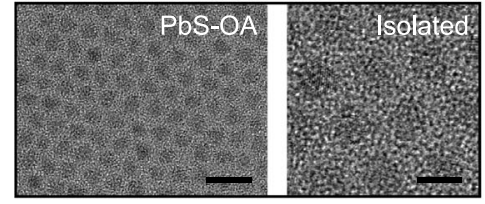

b

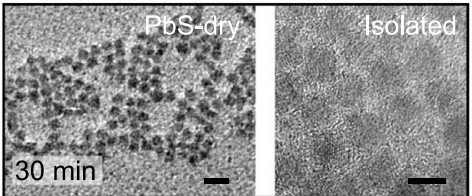

C

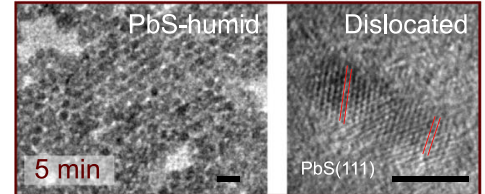

d

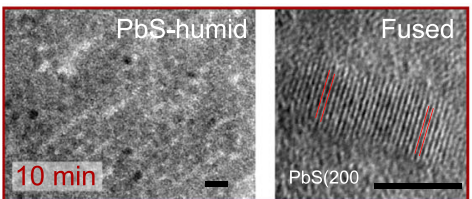

e

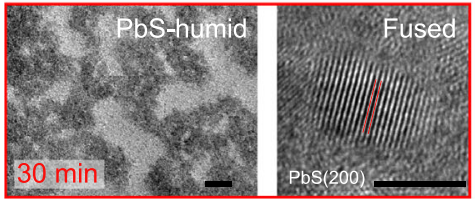

f Dry condition

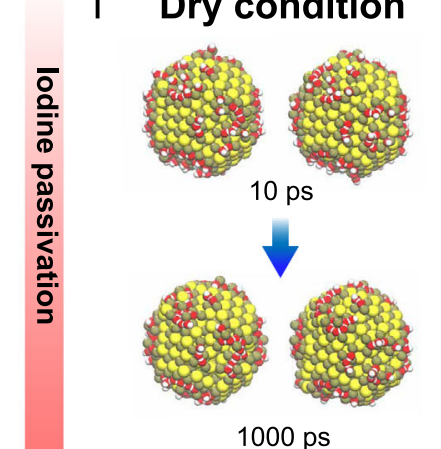

g Humid condition

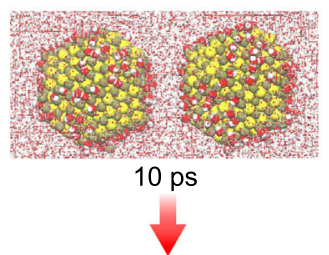

\section{Close attached}

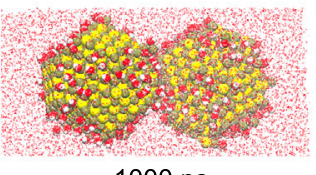

1000 ps $\mathrm{h}$
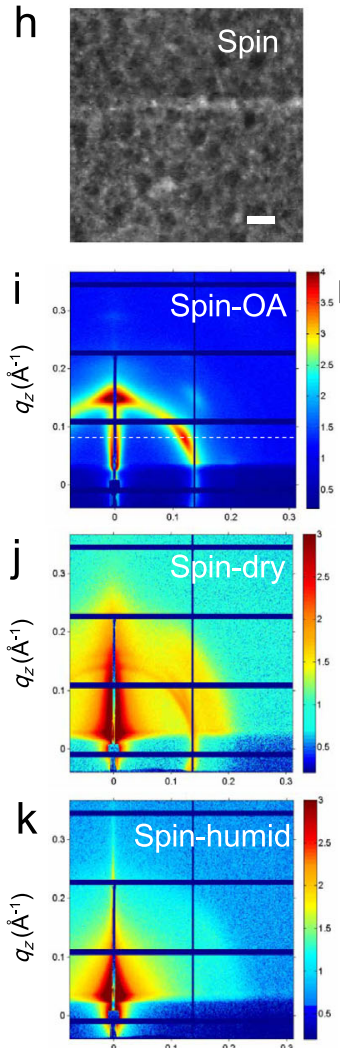

$q_{y}\left(\AA^{-}-1\right)$
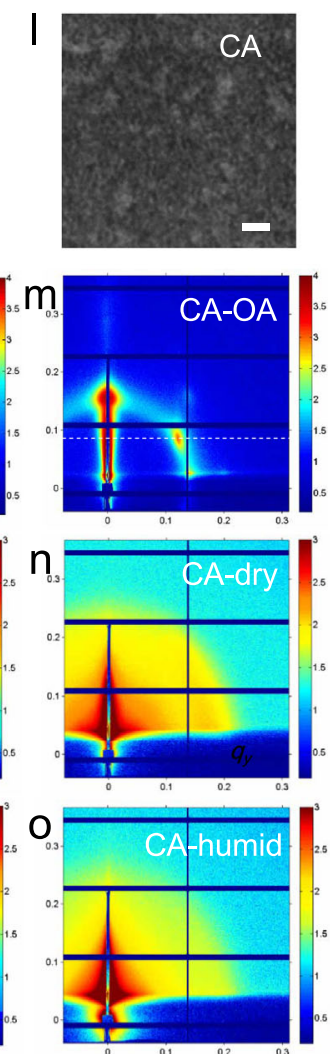

$q_{y}\left(\AA^{-1}\right)$

p Meniscus-guided coating

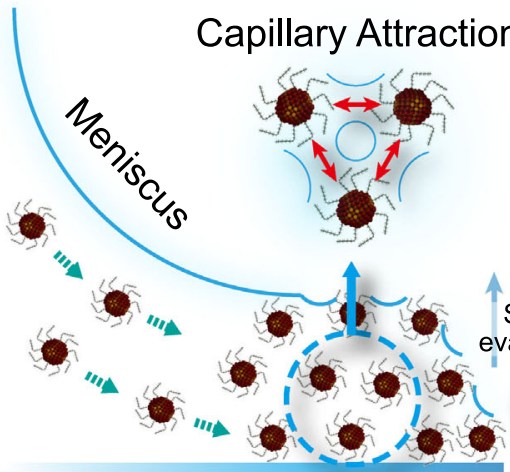

Capillary Attraction

Cracks and pinholes

\section{Spin coating}

CQDs fusion
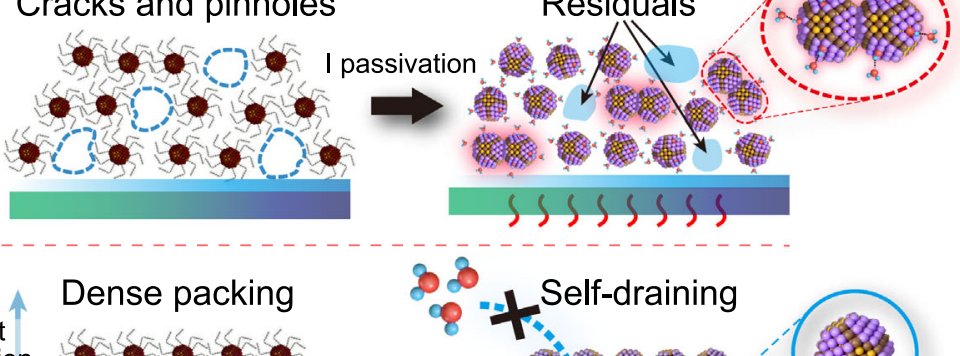

Solvent
evaporation

I passivation
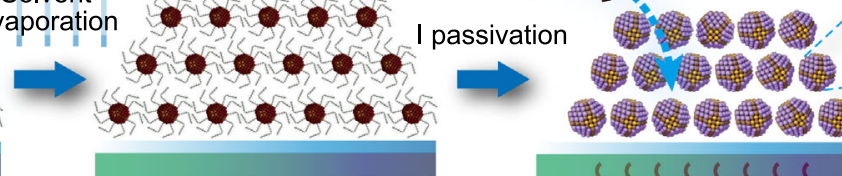

cose
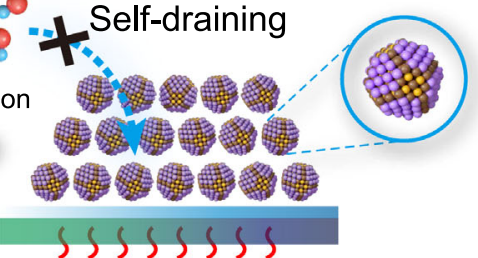

Fig. 2 The impact of ambient water on the CQD nanostructure and film morphology. a-e The TEM images of PbS CQDs prepared under different conditions. The samples were prepared on carbon meshes by (a) droplet deposition (b) followed by ligand exchange and annealing process at $85^{\circ} \mathrm{C}$ under dry air, (c-e) or under humid air with different annealing times. The scale bars: left column $10 \mathrm{~nm}$ and right column $5 \mathrm{~nm}$. $\mathbf{f}, \mathbf{g}$ Molecular dynamic simulation of the water effect on hydroxylated CQDs. Atom color: brown, Pb; yellow, S; red, O; white, H. Snapshots of two CQDs with surface hydroxyls covered on $\{111\}$ facet with a gap distance of $1 \mathrm{~nm}$ at $300 \mathrm{~K}$ after (f) $10 \mathrm{ps}$ and (j) $1000 \mathrm{ps}$. The ambient water could work as H-bonding bridges, which significantly promote the close attachment of neighboring CQDs. h, I The focused ion beam (FIB) cross-sectioned bright-field TEM images of CQD stacks prepared through (h) spin coating and (I) CA. Scale bars: $10 \mathrm{~nm}$. The GISAXS patterns of CQD films prepared by (i) spin coating before ligand exchange and (j) after ligand exchange under dry air annealing or ( $(\mathbf{k})$ under humid air annealing, or by ( $\mathbf{m}$ ) CA deposition before ligand exchange and (n) after ligand exchange under dry air annealing or (o) under humid air annealing. $\mathbf{p}$ The illustration of meniscus-guided coating and spin-coating process for the preparation of PbS CQD solids. The densely packed CA films show the self-draining effect against ambient water intrusion.

Generally, the CQDs should go through several steps before completely fusing into large crystals, including the CQD close attachment, rotations, planar alignments, and interfacial relaxation ${ }^{43}$. This process can be affected by multiple factors, including the van der Waals (vdW) attractions, entropic factors, and the atomic bonding of specific crystal facets ${ }^{44}$. In any case, the close attachment of neighboring CQDs is the prerequisite for fusion processing. We suppose that the water molecules displaced between hydroxylated CQD surfaces could work as $\mathrm{H}$-bonding bridges, which provide extra inter-CQD attractions and thus facilitates CQD fusion. To testify our hypothesis, molecular dynamic (MD) simulations are performed and shown in Fig. 2f, g. 

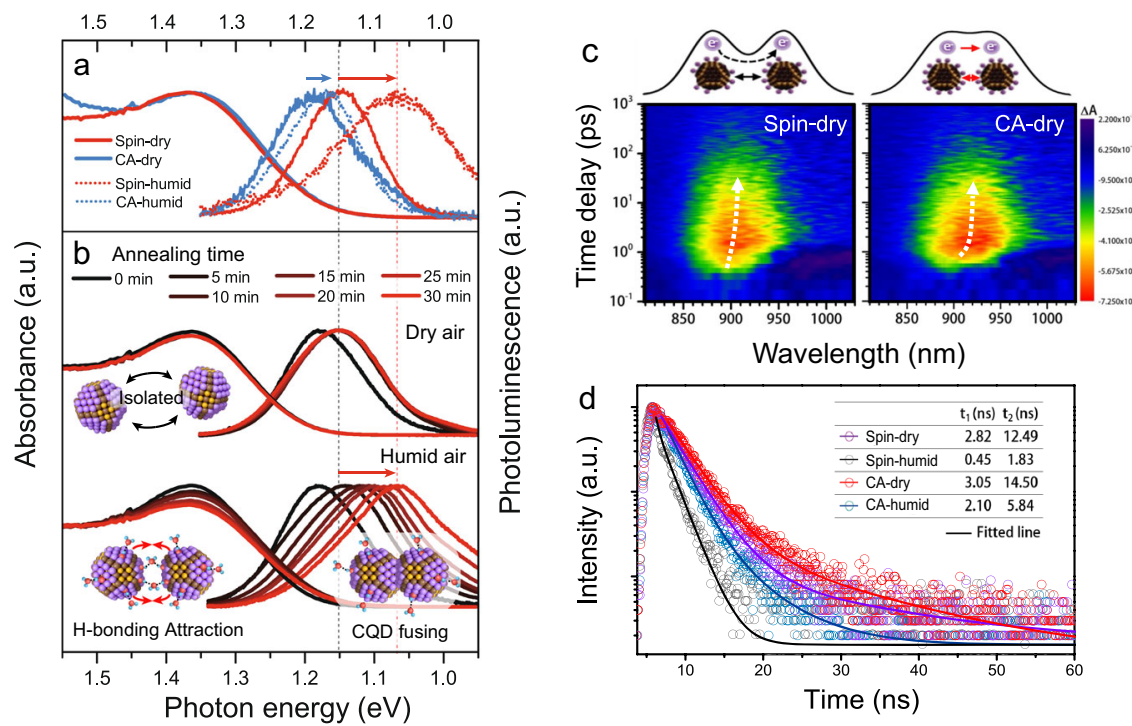

Fig. 3 Spectroscopy and carrier dynamic studies of the CQD solids impacted by ambient water. a The absorbance, steady-state photoluminescence (PL) spectra of PbS-I CQD films prepared by spin coating and CA under different humidity. $\mathbf{b}$ The change of absorbance and PL spectra of spin-coated PbS-I CQD films versus heating time under dry and humid air. c Pseudo-color TA spectra of PbS-I CQD films by spin coating and CA. The color scales for a spin and CA films are the same and proportional to the absorption change in each graph. All samples were pumped by $470 \mathrm{~nm}$ laser pulse with the pump flux of $25 \mathrm{~J} \cdot \mathrm{cm}^{-2}$. d Transient PL decay of CQD films; inset table: the fast and slow time component fitted by double-exponential function.

Two individual hydroxylated CQDs are displaced with a gap distance of $1 \mathrm{~nm}$ at $300 \mathrm{~K}$ in the first place. After $1000 \mathrm{ps}$, almost no change in inter-CQD distance is found for the condition without ambient water. In sharp contrast, the CQDs under humid ambient rapidly attach with each other, which highlights the essential role of ambient water in CQD fusion. To understand the possible driving force for the close attachment assisted by water, we performed DFT calculations on face-to-face $\mathrm{PbS}\{111\}$ slabs with different passivation conditions, as shown in Supplementary Fig. 9. By replacing half of the surface iodine with hydroxyls bonded by ambient water, the relaxed inter-facet distance is remarkably reduced from $4.216 \AA$ to $3.776 \AA$. The $\mathrm{O}$ in $\mathrm{H}_{2} \mathrm{O}$ could form two $\mathrm{H}$-bonds with surface hydroxyls on neighboring $\{111\}$ facets, linking the CQD together and promoting CQD close attachment. This result fits well with the peak shift observed in the XAS spectra (Fig. 1b), where the formation of the H-bonding bridges between neighboring CQDs may promote the transformation of adsorbed surface water from a single donor to double donor configuration, shifting the peak edge to higher energy. The closely attached CQDs have certain mobility under gentle annealing (far below the melting point of bulk crystals), promoting further rotations and fusion along $\{100\}$ facet ${ }^{43,45}$.

In addition, we find that the morphology-governed water entrapping in $\mathrm{PbS}$ CQD solids can be affected by different deposition techniques. The traditional SSLE PbS-I CQD films spin-coated by layer-by-layer (LbL) method inevitably generate inhomogeneous film morphology with striation structures, as shown in the cross-sectional TEM images in Fig. $2 \mathrm{~h}$ and Supplementary Fig. 10. The strong black-and-white contrast in the TEM image corresponds to the difference of electron scattering throughout the cross-sectional CQD slide with a thickness of $\sim 100 \mathrm{~nm}$, reflecting incompact bulk morphology inside CQD films ${ }^{46,47}$. The porous morphology in CQD solids may enhance water trapping and CQD surface hydroxylation. To reduce the impact of ambient water, a meniscus-guided-coating technique, i.e., convective assembly (CA) is introduced ${ }^{48}$. Benefiting from the strong capillary attraction among CQDs during the assembly process, a much more compact film can be achieved without apparent voids and organic striations (Fig. 2l), which reduces water preservation in CA films. To further understand the effect of water on film morphology at different spatial scales, the GISAXS patterns of CQD films fabricated under different conditions were collected and presented in Fig. 2i-k and Fig. $2 \mathrm{~m}-\mathrm{o}$, with the relative azimuthally integrated patterns exhibited in Supplementary Fig. 11. The OA capped CQD films before ligand exchange shows a distinct diffraction ring, indicating an inter-CQD spacing of $5.18 \mathrm{~nm}$ (Fig. $2 \mathrm{~m}$ ) and 5.35 $\mathrm{nm}$ (Fig. 2i) for CA film and spin-coated film, respectively ${ }^{49,50}$ The shorter inter-dot spacing demonstrates the superior compactness of CA film. Ligand exchange reduces the packing order and broadens the diffraction pattern for both PbS-I films. The mingled rings in Fig. $2 \mathrm{j}$ indicate inter-spaces between 5.35 and $5.43 \mathrm{~nm}$, likely due to the incompletely exchanged OA ligands or other organic residuals in spin-coated film. In comparison, uniform diffraction patterns can be observed in Fig. 2n confirming the improved homogeneity for close-packed CA film. More importantly, the morphology of CQD solids is found to be sensitive to ambient water. After annealing for $30 \mathrm{~min}$ under humid air, the GISAXs spectrum of spin-coated PbS-I films (Fig. 2k) changes significantly. The weak diffraction pattern suggests less ordered CQDs packing, where the inter-space is mainly governed by the large inter-domain distance between fused CQDs aggregations, as reflected by the TEM images in Fig. $2 \mathrm{~h}$, and illustrated in Fig. $2 \mathrm{p}$ (right). In sharp contrast, the pattern of CA film with stable surface morphology (Supplementary Fig. 12) shows no significant change towards different ambient RH (Fig. $2 \mathrm{n}-\mathrm{o}$ ), exhibiting strong water resistance. The inherent advantages of meniscus-guided assembly give rise to the self-draining effect to effectively extrude water residuals in CQD films, as illustrated in Fig. 2p (left), which minimizes the subsequent hydroxylation and water adsorption on the CQD surface as proved by the XPS (Supplementary Fig. 5e) and the XAS (Supplementary Fig. 13) results.

Water impacted photoelectric properties of CQD solids. The ambient water significantly affects the PbS CQD surface chemistry and morphology, consequently, changing the optical properties and carrier dynamics in CQD films. Figure 3a shows the 
Table 1 Summarized photoelectric properties for the CQD solids prepared under different conditions.

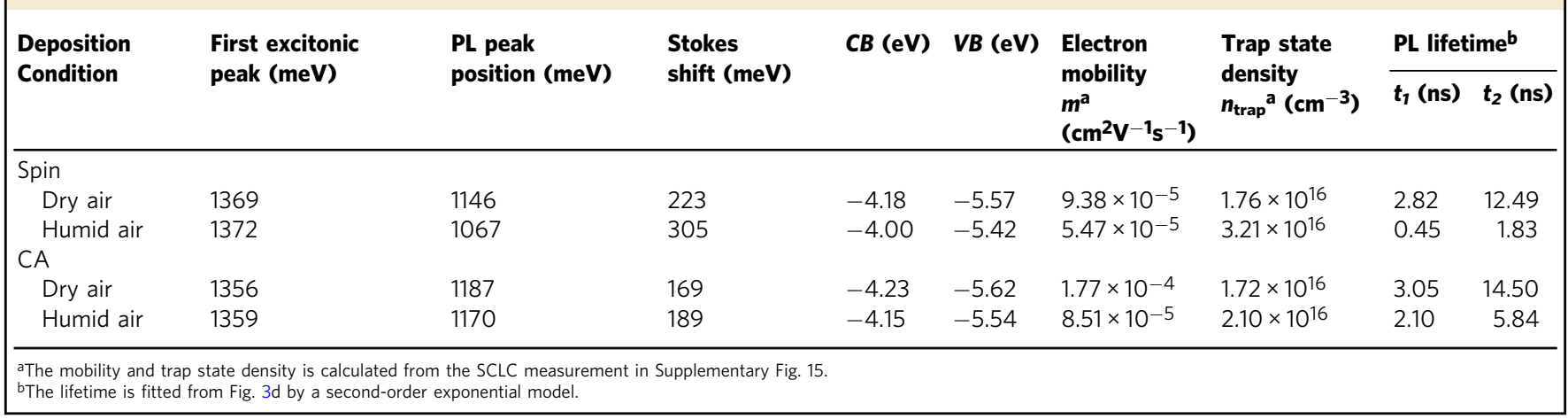

absorption and photoluminescence (PL) spectra of PbS-I films prepared under different conditions followed by annealing at $85^{\circ} \mathrm{C}$ for $30 \mathrm{~min}$. The corresponding optical parameters are summarized in Table 1. A Stokes shift of $169 \mathrm{meV}$ for the CA film prepared under dry air can be observed, while the spin-coated film shows a Stokes shift of $223 \mathrm{meV}$. The smaller Stokes shift can be ascribed to the better CQDs packing order in CA film, leading to flattened energetic landscape and improved charge carrier transport ${ }^{51-53}$. Resultantly, the CA film shows a rapid spectra diffusion in transient absorption spectra (TAS) at the time scale of several picoseconds (Fig. 3c, Supplementary Fig. 14), indicating the fast charge transfer due to enhanced electrical coupling in ordered CQD arrays (see Supplementary Note 1 for detailed explanations). In comparison, the inhomogeneous morphology of spin-coated film causes an uneven energetic landscape, impeding the charge transport and broadening the Stokes shift to a certain extent ${ }^{54,55}$. After exposure of the fabrication process to humid ambient (RH 50-60\%), a drastically increased Stokes shift of 305 $\mathrm{meV}$ can be observed for the spin-coated film. In contrast, a Stokes shift of only $189 \mathrm{meV}$ is observed for CA film, with a slight change of $20 \mathrm{meV}$ induced by humidity variation. Note that in the previous studies, the surface vacancies in PbS CQD have been widely regarded as the source of trap states, which could vary the Stokes shift to more than hundreds of $\mathrm{meV}^{36,56,57}$. However, in our DFT results, the introduction of the surface hydroxylation or water adsorption does not reduce the vacancy formation energy of surface iodine ( $E_{\mathrm{vac}-\mathrm{I}}$ from $3.31 \mathrm{eV}$ to $3.38 \mathrm{eV}$ on $\{111\}$ facet). Instead, the high vacancy formation energy of $\mathrm{OH}\left(E_{\mathrm{vac}-\mathrm{OH}}\right.$ of $4.77 \mathrm{eV}$ ), as shown in Fig. 1b, makes the CQD surface vacancies thermodynamically hard to be formed ${ }^{34}$. (see Supplementary Note 2 for detailed explanations). Thus, a new mechanism may lie behind the discrepancy.

Our studies reveal that the Stokes shift is strongly correlated with the extent of CQD fusion and the corresponding hydroxylates amount on CQD surfaces. Figure $3 \mathrm{~b}$ shows the absorbance and PL spectra of spin-coated PbS-I CQD films annealed under different ambient conditions. The films annealed under dry air exhibit a very limited peak shift in PL spectra, where the individual CQDs remained to be well-isolated (Fig. 2b). As a comparison, for the film heated under humid air, the PL peak red-shifts continuously with increasing annealing time, accompanied by the diminution of the first excitonic absorption peaks. This change in spectroscopy is well-fitted to the continuously intensified CQD fusion aided by ambient water as observed in Fig. 2c-e. The strong electrical coupling between fused CQDs generates new states lower in energy by $100-200 \mathrm{meV}$ relative to the CQD bandgap ${ }^{58-60}$, creating inter-band traps that broaden the Stokes shift and reduce the PL lifetime (Fig. 3d) ${ }^{61}$. This result is well in accordance with the enhanced trap-filled limited voltage $\left(V_{\mathrm{TFL}}\right)$ and trap states density $\left(n_{\text {trap }}\right)$ from the space-charge- limited current (SCLC) measurements in Supplementary Fig. 15. Thus, we owe the large Stokes shift in spin-coated CQD film under humid conditions to the serious CQD fusion, rather than the formation of surface Schottky vacancies. We found that the water-triggered fusion and related trap behavior in CQD devices are very general. The effect of water on atomic halide passivated CQD active layers prepared by three different methods are studied, including the solution-phase-ligand-exchanged (SPLE) inks and the direct-synthesized (DS) inks of PbS CQDs, as well as the SPLE PbSe CQD inks, as shown in Supplementary Fig. 16. Similar trends in surface conditions and optical properties can be concluded, exhibiting the generality of water-triggered fusion and related trap behavior in atomic halide passivated CQDs solids.

Water affected solar cell performance and thermal stability. The surface conditions, film morphology, and carrier dynamics in CQD solids altered by ambient water would eventually determine the photovoltaic performance. Figure 4a shows the solar cell architecture and device performance. The band-gap diagram of solar cells at the working conditions and the energy level of each functional layer are presented in Fig. 4c-e and Supplementary Fig. 17. The detailed device parameters are summarized in Table 2. For the solar cells prepared under dry air, the CA devices yield a well-matched energy level alignment as shown in Fig. 4c, giving an average power conversion efficiency (PCE) of $11.2 \%$ with a remarkable FF of $69.5 \%$. A champion PCE of $11.6 \%$ was achieved, which is so far the best reported CQD solar cells prepared by meniscus-guided-coating techniques. The fast charge transport as proved by the rapid spectra diffusion in TAS and higher electron mobility in Table 1 , as well as the longest electron recombination lifetime ( $\tau_{r}$, Fig. $4 \mathrm{~b}$, Supplementary Fig. 18) account for the efficient charge collection and thus high device FF. The spin-coated cells prepared under dry air show an average PCE of $10.2 \%$ with a moderate FF of $63.7 \%$. The morphology defects with the significant appearance of organic residuals in CQD solids may harm the charge collection and device FF (Fig. 4c), which can be evidenced by the small geometric capacitance $\left(C_{\text {geo }}\right)$ and large transport resistance $\left(R_{\text {tr }}\right)$ from the electrochemical impedance spectroscopy (EIS) in spin-coated cells (Supplementary Fig. 19, Table S6) ${ }^{62}$.

Under humid conditions, serious CQD fusion causes interband traps and inhomogeneous energetic landscape, limiting the charge transport and $V_{o c}$ in solar cells. Meanwhile, the surface hydroxylation reduces the total surface dipole moment, which shifts the band edge of PbS-I CQD layer up to the vacuum level and weakens the electron blocking ability of PbS-EDT layer ${ }^{63}$. The change of energy level is consistent with the DFT simulation in Supplementary Fig. 2d. As a result, increased interface recombination occurs as illustrated in Fig. 4e, reflected by the reduced $\tau_{r}$ and the increased ideality factors $\left(n_{\text {ideal }}\right)$ 

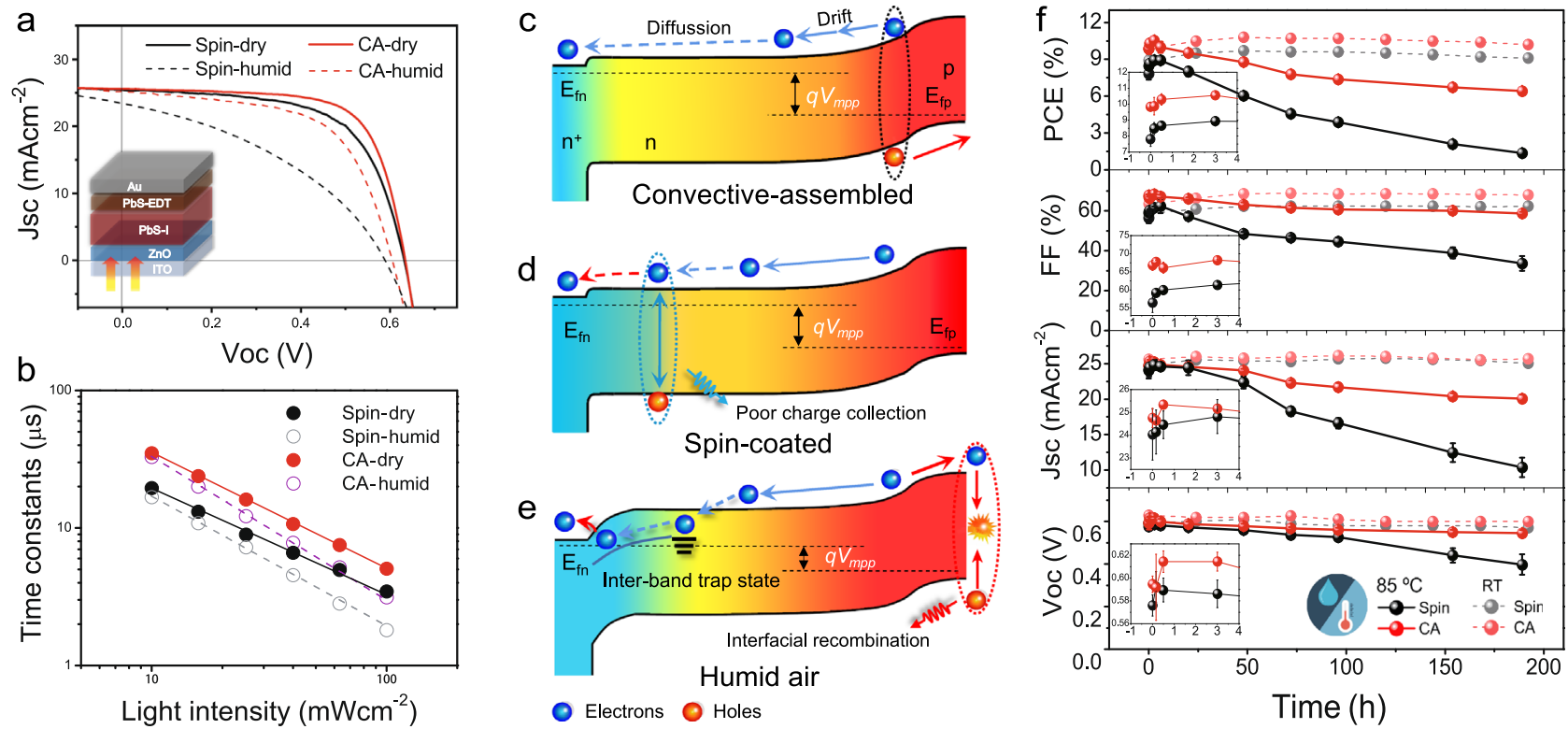

Fig. 4 Solar cell performance of CQD devices intervened by ambient water. a $J-V$ curves of CQD solar cells fabricated through the spin coating and CA under dry air and humid air ( $\mathrm{RH} \sim 50 \%-60 \%$ ), measured under AM $1.5 \mathrm{G}$ solar simulator. b Light-intensity-dependent electron recombination lifetime constants for CQD devices. The relative Bode plots are shown in Supplementary Fig. 18. c-e Band diagrams for CQD cells at $V_{M P P}$ condition for (c) CA devices and (d) spin-coated devices prepared under dry air and (e) under humid air, where $E_{F n}$ is the electron quasi-Fermi level, and $E_{F p}$ is the hole quasiFermi level. $\mathbf{f}$ Stability of CQD devices under room temperature or under continuously heating at $85^{\circ} \mathrm{C}$ in ambient air. The parameters evolutions within the early hours are inserted in each graph.

(Supplementary Fig. 20, Table 2) for devices prepared under humid air. In addition, the Fermi-level shifts down in spin-coated CQD films under humid air, which leads to a lower built-in potential $\left(V_{\mathrm{bi}}\right)$ (Supplementary Fig. 21, Table 2). Resultantly, the humid ambient results in inferior device performance for the spin-coated devices, due to fusion-induced inter-band traps and mismatched energy levels in solar cells. The average PCE drastically drops from $10.2 \%$ to $5.2 \%$ with the FF reduced from 63.7 to $39.0 \%$. The adverse effects of ambient water on the CQD devices prepared by different CQD inks are also investigated in Supplementary Fig. 22g-i. Fortunately, benefiting from the selfdraining effect, the photovoltaic performance of CA devices is much less affected by humid air, still offering a decent PCE of $10.0 \%$.

To exam, the possible influence of ambient water on device stability, the aging of CQD solar cells accelerated by continuous heating at $85^{\circ} \mathrm{C}$ under humid ambient ( $\mathrm{RH} \sim 50-60 \%$ ) is monitored in Fig. 4f. The devices exhibit fast gains in PCE within several minutes ${ }^{64}$, followed by continuous decays in FF and PCE during the subsequent heating period. CA devices exhibit apparently better thermal stability than spin-coated ones. However, the decay is still significantly faster than the devices stored at room temperature. To elucidate that, the XPS of PbS-I CQD film exposed to humid air under continuous heating is collected and shown in Supplementary Fig. 6b-d. The long-time surface hydroxylation massively shrinks the iodine coverage on CQD surfaces, following the reaction in Eq. 4. In addition to the serious surface I loss, the byproduct of $\mathrm{I}_{2}$ gas may lead to significant iodine migrations in CQD devices, especially in spincoated ones, as shown in the STEM-EDX images (Supplementary Fig. 23). The I migration could damage the buffer layers and electrodes, harming the charge extraction reflected by incessantly dropped FF and $J_{\mathrm{sc}}$ in solar cells ${ }^{65}$. Moreover, the enhanced fusion with continuous heating under humid air will inevitably cause an uneven energetic landscape, eventually reducing device performance and stability. It is worth mentioning that protecting CQD devices from the ambient humidity by device encapsulation can decelerate the decay, while the entrapped water inside the film, as evidenced by the enhanced O1s signals in Supplementary Fig. $5 c$, will still lead to device degradation under annealing (Supplementary Fig. 24). In short, we attribute the performance decay under humid ambient with annealing to the iodine loss and migration during the hydroxylation process, as well as related aggravation of CQD fusion. This revealed effect of ambient water may provide a clearer clue for the understanding of the experimental observations on trap behaviors, device deficiency, and instability in lead chalcogenide CQD electronics involving hydroxyl issue $16,17,19,21,23,66-68$.

\section{Discussion}

In summary, we identified that the hydroxylated PbS CQD surfaces are covered by $\mathrm{H}$-bonded water, which governs the temperature-dependent evolution of the surface chemical environment and generates significant effects on CQD nanostructure morphology, optoelectronic properties, as well as final photovoltaic performance. The entrapped water in CQD solids enhances the surface hydroxylation, aiding the fusion of neighboring CQDs in spin-coated film. Consequently, the disordered nanostructure morphology leads to inhomogeneous energetic landscapes and inter-band trap states, resulting in significantly broadened Stokes shift and reduced device performance. Moreover, iodine loss and migration during the hydroxylation process were revealed as the key factors for the poor thermal stability of $\mathrm{PbS}$ CQD solar cells. To address these issues, convective assembly was utilized for the fabrication of CQD solids. The improved homogeneity and compactness in CQD films introduce a self-draining effect to alleviate ambient water intrusion, leading to suppressed surface hydroxylation and fusion. Our result will shed light on the fundamental understanding of waterinvolved CQD surface chemistry and its effect on CQDs properties and photovoltaic performance, which may generate a farreaching influence on the ambient scalable fabrication of CQD based electronics. 
Table 2 Summarized photovoltaic parameters for the CQD solar cells prepared under different conditions.

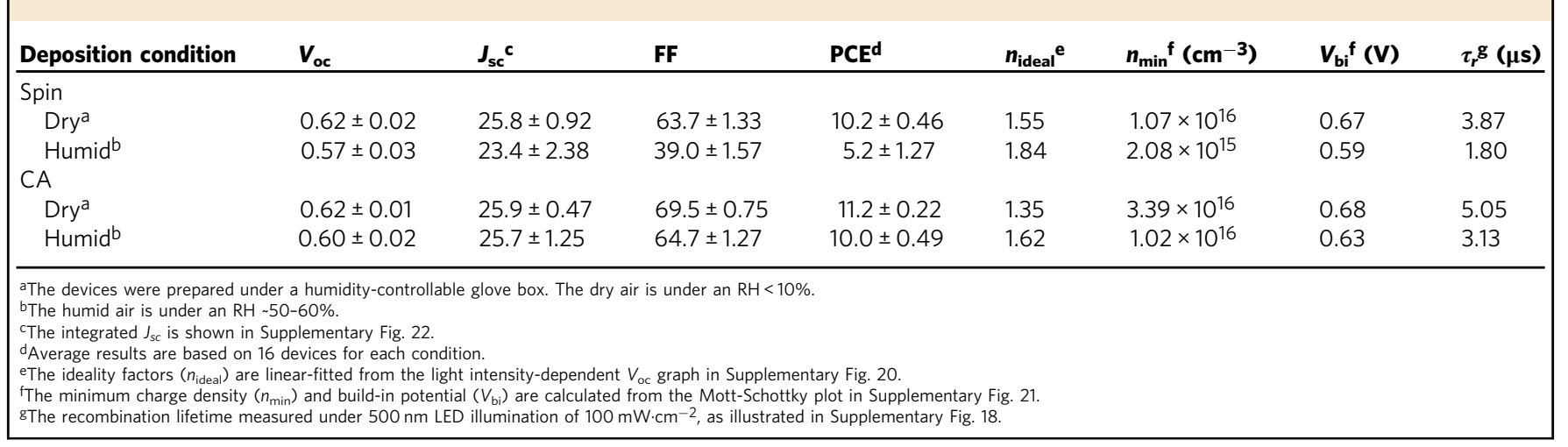

\section{Methods}

Synthesis of PbS CQDs. All operations were performed under a nitrogen atmosphere using standard air-free Schlenk line techniques. For the synthesis of $\sim 3 \mathrm{~nm}$ PbS CQDs. A solution of $380 \mathrm{mg}$ of lead acetate trihydrate $(1 \mathrm{mmol}), 0.7 \mathrm{~g}$ of oleic acid $(2.5 \mathrm{mmol})$, and $20 \mathrm{~g}$ of 1 -Octadecene (ODE) was degassed at $100^{\circ} \mathrm{C}$ in a 100 $\mathrm{ml}$ three-neck flask for $1 \mathrm{~h}$ under vacuum. The solution was then heated for an additional $1 \mathrm{~h}$ to $150^{\circ} \mathrm{C}$ under nitrogen. After adjusting the solution to the desired temperature, $0.5 \mathrm{mmol}$ hexamethyldisilathiane ((TMS) $\left.)_{2} \mathrm{~S}\right)$ dissolved in $5 \mathrm{ml} \mathrm{ODE}$ was rapidly injected into this hot solution. The CQDs were grown at $80^{\circ} \mathrm{C}$ for the optimal time, and the reaction was rapidly quenched by placing the flask in a room-temperature water bath and injecting $8 \mathrm{ml}$ of anhydrous hexane, then purified by precipitation in hexane and isopropyl alcohol and once in hexane/acetone and stored with solid form in a nitrogen-filled glove box.

Photoemission spectroscopy (PES). Ultra-violet photoelectron spectroscopy (UPS) and X-ray photoelectron spectroscopy (XPS) were carried out in an ultrahigh vacuum (UHV) system with a base pressure of $1.0 \times 10^{-10} \mathrm{mbar}$. To avoid water adsorption during the transfer from the glove box to UHV, the samples were transferred in a transfer box filled with ultra-pure $\mathrm{N}_{2}$ gas to the load-lock chamber of the UHV system with a base pressure of $1.0 \times 10^{-7} \mathrm{mbar}$. XPS was measured with a monochromatized $\mathrm{Al} \mathrm{Ka}$ light source $(1486.75 \mathrm{eV})$, while for UPS the likewise He I $\alpha$ line $(21.22 \mathrm{eV})$ was used.

X-ray absorption spectroscopy (XAS). The O K-edge XAS spectra were measured at beamline 20A1 of Taiwan. The PbS CQD samples were prepared on top of a silicon wafer under different conditions. The commercial $\mathrm{Pb}(\mathrm{OH})_{2}, \mathrm{PbO}$ powders were compressed into films on top of Au wafers by a tablet machine. All samples were transferred under the $\mathrm{N}_{2}$ box. Before measurements, the samples were first mounted onto the sample holders in a special glovebox attached to the load-lock chamber and then transferred to the analysis chamber under ultrahigh vacuum conditions. The O K-edge XAS spectra in total-electron-yield (TEY) mode was first normalized to the beam flux measured by a gold mesh and then normalized to the absorption pre-edge and post-edge. The photon energy was calibrated by the reference O K-edge spectra of $\mathrm{SrTiO}_{3}$.

Density functional theory (DFT). All density functional calculations were performed by using VASP code ${ }^{69}$. The electronic structures of the system were calculated with the Perdew-Burke-Ernzerhof revised for solids (PBEsol) functional and the projector-augmented wave (PAW) method under cut-off energy of $800 \mathrm{eV}$. The $(5 \mathrm{~d})^{10}(5 s)^{2}(6 s)^{2}(6 p)^{2}$ valence electrons of lead, $(3 s)^{2}(3 p)^{4}$ valence electrons of sulfur, $(5 s)^{2}(5 p)^{5}$ valence electrons of iodine, $(2 s)^{2}(2 p)^{4}$ valence electrons of oxygen and $(1 \mathrm{~s})^{1}$ electron of hydrogen were considered in the calculations. $4 \times 4 \times$ 4 and $4 \times 4 \times 1 \Gamma$ centered $k$-points sampling of the Brillouin zone were used for optimizing both the cell parameters and the atomic coordinates of the bulk and slab structures, respectively. The optimized lattice parameters of the (111) oriented bulk $\mathrm{Pb}_{12} \mathrm{~S}_{12}$ structure were $10.22 \AA, 7.23 \AA$, and $8.35 \AA$ for $\mathrm{A}, \mathrm{B}, \mathrm{C}$, respectively. The lattice parameter of the (111) oriented slab was $100 \AA$ for A and the B and C parameters of them were kept to those of the bulk structure. The surface energy $\gamma$ is calculated as: $\gamma=\left[E\left(\mathrm{nPbS} / \mathrm{mPbL}_{2}\right)-\mathrm{nE}(\mathrm{PbS})-\mathrm{mE}(\mathrm{PbL})\right] / A$, where $E(\mathrm{X})$ is the total energy of the system $\mathrm{X}, A$ is surface area, $\mathrm{n}$ and $\mathrm{m}$ represent the numbers of $\mathrm{PbS}$ and $\mathrm{PbL}$ units in the system, respectively, and $\mathrm{L}$ represents the capping ligands such as iodide, $\mathrm{OH}$ group, and oxygen anion. The total energies of $\mathrm{PbS}$ and $\mathrm{PbL}$ were taken from bulk $\mathrm{PbS}$ and $\mathrm{PbL}$ precursor molecules, respectively. The formation energy of the surface vacancy of the radical species is calculated as (the details are summarized in Supplementary Table 2):

$$
E_{\text {vac }}=\left[\mathrm{E}\left(\mathrm{Pb}_{\mathrm{x}} \mathrm{S}_{\mathrm{y}} \mathrm{L}_{\mathrm{z}}\right)-\left(\mathrm{E}\left(\mathrm{Pb}_{\mathrm{x}} \mathrm{S}_{\mathrm{y}} \mathrm{L}_{\mathrm{z}-2}\right)+2 \mathrm{E}(\mathrm{L})\right)\right] / 2
$$

Fermi energy term is not considered in this calculation because all $\mathrm{Pb}_{x} \mathrm{~S}_{y} \mathrm{~L}_{z}$, $\mathrm{Pb}_{\mathrm{x}} \mathrm{S}_{y} \mathrm{~L}_{z-2}$, and $\mathrm{L}$ are electrically neutral because $\mathrm{L}$ is radical species. The water adsorption energy is calculated as:

$$
E_{\mathrm{ad}}=\left[\left(\mathrm{E}\left(\mathrm{Pb}_{\mathrm{x}} \mathrm{S}_{\mathrm{y}} \mathrm{L}_{\mathrm{z}}\right)+2 \mathrm{E}\left(\mathrm{H}_{2} \mathrm{O}\right)\right)-\mathrm{E}\left(\mathrm{Pb}_{\mathrm{x}} \mathrm{S}_{\mathrm{y}} \mathrm{L}_{\mathrm{z}}+2 \mathrm{H}_{2} \mathrm{O}\right)\right] / 2
$$

The density of states (DOS) is shifted to align the vacuum level to zero.

Fabrication of CQD solar cells. $\mathrm{ZnO}$ nanoparticles were synthesized and prepared according to the reported method ${ }^{18}$. The $\mathrm{ZnO}$ NCs solution was then spin-coated on a clean ITO substrate at $2500 \mathrm{rpm}$ for $30 \mathrm{~s}$. The iodine passivated CQD active layer was prepared by the layer-by-layer (LbL) method.

Spin coating. The solid-phase-ligand-exchanged PbS-I CQD stakes were prepared by spinning coating $100 \mu \mathrm{L} \mathrm{PbS} \mathrm{CQDs} \mathrm{from} 40 \mathrm{mg} \mathrm{mL}^{-1}$ hexane solution at 2500 $\mathrm{rpm}$. The ligand exchange was carried out by dropwise coating the TBAI $10 \mathrm{mg} \mathrm{ml}$ ${ }^{-1}$ tetrabutylammonium iodide (TBAI) methanol solution for $30 \mathrm{~s}$ on as-prepared films, and then rising with pure methanol followed through washing with acetonitrile and drying by a nitrogen gun. This procedure was repeated 6 times to achieve a nearly $250 \mathrm{~nm}$ thickness. The humidity was controlled from 10 to $60 \%$ by a humidifier within the glove box. The humid condition was applied with an RH of $\sim 50-60 \%$ unless specifically mentioned. The dry air is using compressed air gas with an $\mathrm{RH}$ of less than $10 \%$.

Convective assembly. Ten microliters of the CQDs solution was placed between the glass blade and ITO substrate which keeping an angle of $45^{\circ}$. A stepper motor was used to recede the substrate with uniform velocity. Different conditions were optimized according to a microscopic model proposed by Mae"l Le Berre et al. ${ }^{70}$ The optimized deposition conditions were using hexane as CQDs solvent with a concentration of $80 \mathrm{mg} / \mathrm{ml}$ and a receding speed of $1 \mathrm{~mm} \mathrm{~s}^{-1}$. After ligand exchange, acetonitrile was dropwise added on top of the substrate followed by quick drying through a nitrogen gun. The average thickness of each deposition cycle is around $30 \mathrm{~nm}$. This procedure was repeated to achieve a nearly $250 \mathrm{~nm}$ thickness. After the deposition of PbS-I CQD layers, $20 \mathrm{mg} \mathrm{ml}^{-1} \mathrm{PbS}$ deposited on top of PbS-I layers followed by an EDT solution ( $0.04 \mathrm{vol} \%$ in acetonitrile) treatment for $30 \mathrm{~s}$ and acetonitrile rinsing twice. This procedure was repeated twice to get a thickness of $40 \mathrm{~nm}$. The prepared devices were stored under dry air overnight for further oxidation and sequentially annealed at $85^{\circ} \mathrm{C}$ for $30 \mathrm{~min}$ before the evaporation of the gold electrode. Finally, a $100 \mathrm{~nm}$ Au layer $\left(0.5 \AA \mathrm{s}^{-1}\right)$ was evaporated through thermal evaporation under a high vacuum lower than $1 \times 10^{-5}$ mbar through a shadow mask to define a total active area of $0.0725 \mathrm{~cm}^{2}$. The solution-phase ligand exchange of $\mathrm{PbS} C \mathrm{CQD}$ and the direct synthesis of $\mathrm{PbS} C \mathrm{CQD}$ inks were performed according to our previous reports ${ }^{18,71}$.

Measurements and characterizations. Current density-voltage $(J-V)$ characteristics of the devices were measured under ambient air using a programmable Keithley 2401 source meter under a simulated AM 1.5 G solar irradiation of 100 $\mathrm{mWcm}^{-2}$. (Newport, Class AAA solar simulator, 94023A-U) The light intensity is calibrated by a certified Oriel Reference Cell $(91150 \mathrm{~V})$ and verified with an NREL calibrated Hamamatsu S1787-04 diode. A metal mask was used for defining the active area $\left(0.725 \mathrm{~cm}^{2}\right)$. Voltage swept from $-0.8 \mathrm{~V}$ to $0.8 \mathrm{~V}$ with a speed of $0.01 \mathrm{~V}$ per point and a dwell time of $10 \mathrm{~ms}$. Atomic force microscopy (AFM) images were obtained using a Veeco Multimode V instrument in tapping mode. As for the crosssection TEM and images, the thin film samples were prepared by FIB after carbon coating. The TEM was operated with JEOL JEM-2010FEF at $200 \mathrm{kV}$. To monitor the evolution of CQD nanostructures affected by ambient conditions, the CQD samples in Fig. 2a-e were prepared by dropping the CQDs octane solution $(2 \mathrm{mg} /$ $\mathrm{ml}$ ) on carbon meshes. Under slow evaporation of the solvent, a single-layered 
CQD superlattice structure can be obtained (Fig. 2a). The carbon meshes were then dipped into ligand solutions for the ligand exchange process and put on a hot plate for further heating under dry or humid conditions. The UV-vis-NIR spectra of CQD films were recorded on a Perkin Elmer model Lambda 950 under reflection mode. The steady-state photoluminescence and time-resolved luminescence spectra were measured by exciting the PbS CQD films on quartz substrate at room temperature with a pulsed Nd: YAG laser, using the frequency-doubled line at $531 \mathrm{~nm}$. The luminescence was detected with a Hamamatsu R5509-73 photomultiplier. The photoluminescence lifetimes were measured using a time-correlated single-photon counting system (Hamamatsu C7990). The transient absorption spectroscopy (TAS) was carried out on an fs-TA system. The laser source was a Ti/sapphire laser (CPA-2010, Clark-MXR Inc.) with a wavelength of $775 \mathrm{~nm}$, a pulse width of $150 \mathrm{fs}$, and a repetition rate of $1 \mathrm{kHz}$. The CQD film was sealed into a transparent quartz cuvette (size: $60 \mathrm{~mm} 10 \mathrm{~mm} 40 \mathrm{~mm}$ ) filled with $\mathrm{N}_{2}$ gas, and all samples were pumped by a $470 \mathrm{~nm}$ laser pulse. The electronic recombination lifetime of the CQD device was captured using the intensity-modulated photovoltage spectroscopy (IMVS) profiling by Zahner IM6 electrochemical workstation, applying a bias of varying intensities of light illumination under open-circuit conditions with a 500 $\mathrm{nm}$ LED and a frequency between $0.25 \mathrm{MHz}$ and $0.05 \mathrm{~Hz}$. The yielded $f_{\text {IMVS }}$ is inversely proportional to the electron recombination time constant $\tau_{r}$ by:

$$
\tau_{r}=\frac{1}{2 \pi f_{\text {IMVS }}}
$$

Where $\tau_{r}$ is the electronic recombination lifetime, $f_{\mathrm{IMVS}}$ is the characteristic frequencies correspond to the peaks in the imaginary part in the Bode plot (see Supplementary Fig. 18)

Space charge limited current was conducted using a device structure of ITO/ $\mathrm{ZnO} / \mathrm{PbS}-\mathrm{I} / \mathrm{ZnO} / \mathrm{Al}$. The electron mobility was calculated according to

$$
J=\frac{9}{8} \cdot \varepsilon \varepsilon_{0} \mu \frac{V^{2}}{d^{3}}
$$

Where $\mu$ is the charge carrier mobility, $d$ is the film thickness, $\varepsilon_{0}$ is the vacuum permittivity, while $\varepsilon$ represents the material's dielectric constant, here we used for 18.7 for PbS-I. The relation between the trap density and the onset voltage of the trap-filled-limit (TFL) regime is:

$$
V_{\mathrm{TFL}}=\frac{e N_{t} d^{2}}{2 \varepsilon \varepsilon_{0}}
$$

where $e$ is the unit charge, $d$ is the film thickness, and $N_{t}$ is the trap state density.

The light-dependent open-circuit voltages were measured under a $500 \mathrm{~nm}$ LED with adjustable illumination from $100 \mathrm{~mW} \mathrm{~cm}^{-2}$ to $1 \mathrm{~mW} \mathrm{~cm}^{-2}$, the light ideality factor is calculated according to

$$
n_{L}=\frac{q}{k_{b} T} \cdot \frac{d V}{d(\ln (L))}
$$

Where $k_{b}$ is the Boltzmann constant, $T$ is the temperature, $L$ is the normalized light intensity.

The molecular dynamic simulations. are based on CQD modules with $\{100\}$ and $\{111\}$ surface facets in truncated octahedral shapes. The $\{111\}$ facets are ended with $\mathrm{Pb}$ atoms and the $-\mathrm{OH}$ groups are attached to $\mathrm{Pb}$ atoms to keep the whole $\mathrm{PbS}$ particle in charge neutral. Molecular dynamics simulations are performed with GROMACS [Comp. Phys. Comm. 91, 43-56 (1995); SoftwareX, 1-2, 19-25 (2015)] software and the interactions between atoms were described by a pair potential including a short-range two-body part and a long-range Coulomb part (Nano Lett. 10, 3966-3971 (2010); Sci. Adv. 2019, 5, eaaw5623)

$$
\mathrm{U}_{i j}\left(r_{i j}\right)=\frac{q_{i} q_{j}}{4 \pi \varepsilon_{0} r_{i j}}+4 \varepsilon_{i j}\left[\left(\frac{\delta_{i j}}{r_{i j}}\right)^{12}-\left(\frac{\delta_{i j}}{r_{i j}}\right)^{6}\right]
$$

The partial charge of $\mathrm{Pb}, \mathrm{S}, \mathrm{O}$, and $\mathrm{H}$ atoms are set as $1.29 \mathrm{e},-1.29 \mathrm{e},-2.0 \mathrm{e}$, and $1.0 \mathrm{e}$, respectively. The LJ coefficients are set as $\sigma_{\mathrm{Pb}}=3.29 \AA, \sigma_{\mathrm{S}}=4.36 \AA, \sigma_{\mathrm{O}}=$ $3.15 \AA, \varepsilon_{\mathrm{Pb}} / k_{\mathrm{B}}=30.0 \mathrm{~K}$, and $\varepsilon_{\mathrm{Se}} / k_{\mathrm{B}}=45.3 \mathrm{~K}, \varepsilon_{\mathrm{O}} / k_{\mathrm{B}}=76.5 \mathrm{~K}$, where $k_{\mathrm{B}}$ is the Boltzmann constant. Note that due to the lack of calculation parameters for $\mathrm{PbS}$, the partial charge of $\mathrm{S}$ and $\mathrm{LJ}$ coefficient of $\sigma_{\mathrm{S}}$ are referred to as the parameter of Se used in MD simulation of similar octahedral PbSe CQD. For $\mathrm{H}$ atoms, only Coulomb interaction is considered and the LJ parameters are set to zero. The widely used TIP3P model for water is adopted [J. Chem. Phys. 79, 926 (1983)]. The Lorentz-Berthelot mixing rules are used to determine the interaction parameters between different atom types.

Reporting summary. Further information on research design is available in the Nature Research Reporting Summary linked to this article.

\section{Data availability}

All necessary data generated or analyzed during this study are included in this published article, and other auxiliary data are available from the corresponding authors upon request.
Received: 18 November 2020; Accepted: 22 June 2021; Published online: 19 July 2021

\section{References}

1. Hass, K. C., Schneider, W. F., Curioni, A. \& Andreoni, W. The chemistry of water on alumina surfaces: reaction dynamics from first principles. Science 282, 265-268 (1998).

2. Wernet, P. et al. The structure of the first coordination shell in liquid water. Science 304, 995-999 (2004).

3. Casalongue, H. S. et al. Direct observation of the oxygenated species during oxygen reduction on a platinum fuel cell cathode. Nat. Commun. 4, 1-6 (2013).

4. Boles, M. A., Ling, D., Hyeon, T. \& Talapin, D. V. Erratum: the surface science of nanocrystals. Nat. Mater. 15, 364 (2016).

5. Wheeler, L. M. et al. Targeted ligand-exchange chemistry on cesium lead halide perovskite quantum dots for high-efficiency photovoltaics. J. Am. Chem. Soc. 140, 10504-10513 (2018).

6. Shi, Z. F. et al. Strategy of solution-processed all-inorganic heterostructure for humidity/temperature-stable perovskite quantum dot light-emitting diodes. ACS Nano. 12, 1462-1472 (2018).

7. Kirmani, A. R. et al. Overcoming the ambient manufacturability-scalabilityperformance bottleneck in colloidal quantum dot photovoltaics. Adv. Mater. 30, e1801661 (2018).

8. Martín-García, B. et al. Reduction of moisture sensitivity of PbS quantum dot solar cells by incorporation of reduced graphene oxide. Sol. Energy Mater. Sol. Cells 183, 1-7 (2018).

9. Sun, B. et al. Monolayer perovskite bridges enable strong quantum dot coupling for efficient solar cells. Joule 4, 1542-1556 (2020).

10. Gong, X. W. et al. Highly efficient quantum dot near-infrared light-emitting diodes. Nat. Photon. 10, 253 (2016).

11. Beygi, H., Sajjadi, S. A., Babakhani, A., Young, J. F. \& van Veggel, F. C. J. M. Surface chemistry of as-synthesized and air-oxidized PbS quantum dots. Appl. Surf. Sci. 457, 1-10 (2018).

12. Bertolotti, F. et al. Crystal symmetry breaking and vacancies in colloidal lead chalcogenide quantum dots. Nat. Mater. 15, 987-994 (2016).

13. Lindan, P. J. D., Harrison, N. M. \& Gillan, M. J. Mixed dissociative and molecular adsorption of water on the rutile (110) surface. Phys. Rev. Lett. 80, 762-765 (1998).

14. Yu, S. Y., Kim, H. \& Koo, J. Y. Extrinsic nature of point defects on the $\mathrm{Si}(001)$ surface: dissociated water molecules. Phys. Rev. Lett. 80, 762-765 (1998).

15. Zherebetskyy, D. et al. Hydroxylation of the surface of PbS nanocrystals passivated with oleic acid. Science 344, 1380-1384 (2014).

16. Wang, Y. et al. In situ passivation for efficient $\mathrm{PbS}$ quantum dot solar cells by precursor engineering. Adv. Mater. 30, e1704871 (2018).

17. Cao, Y., Stavrinadis, A., Lasanta, T., So, D. \& Konstantatos, G. The role of surface passivation for efficient and photostable $\mathrm{PbS}$ quantum dot solar cells. Nat. Energy 1, 1-6 (2016).

18. Wang, Y. et al. Room-temperature direct synthesis of semi-conductive $\mathrm{PbS}$ nanocrystal inks for optoelectronic applications. Nat. Commun. 10, 5136 (2019).

19. Song, J. H., Choi, H., Kim, Y.-H. \& Jeong, S. High performance colloidal quantum dot photovoltaics by controlling protic solvents in ligand exchange. Adv. Energy Mater. 7, 1700301 (2017).

20. $\mathrm{Gu}, \mathrm{M}$. et al. Stable PbS quantum dot ink for efficient solar cells by solutionphase ligand engineering. J. Mater. Chem. A. 7, 15951-15959 (2019).

21. Yang, X. et al. Hydroiodic acid additive enhanced the performance and stability of PbS-QDs solar cells via suppressing hydroxyl ligand. Nano-Micro Lett. 12, 37 (2020).

22. Ding, C. et al. Passivation strategy of reducing both electron and hole trap states for achieving high-efficiency $\mathrm{PbS}$ quantum-dot solar cells with power conversion efficiency over 12\%. ACS Energy Lett. 5, 3224-3236 (2020).

23. Sun, B. et al. Ligand-assisted reconstruction of colloidal quantum dots decreases trap state density. Nano Lett. 20, 3694-3702 (2020).

24. Peters, J. L. et al. Ligand-induced shape transformation of PbSe nanocrystals. Chem. Mater. 29, 4122-4128 (2017).

25. Voznyy, O. et al. A charge-orbital balance picture of doping in colloidal quantum dot solids. ACS Nano. 6, 8448-8455 (2012).

26. Yang, J., Meng, S., Xu, L. \& Wang, E. G. Water adsorption on hydroxylated silica surfaces studied using the density functional theory. Phys. Rev. B. 71, 035413 (2005).

27. Karlberg, G. S. \& Wahnstrom, G. An interaction model for $\mathrm{OH}+\mathrm{H}_{2} \mathrm{O}$-mixed and pure $\mathrm{H}_{2} \mathrm{O}$ overlayers adsorbed on $\mathrm{Pt}(111)$. J. Chem. Phys. 122, 194705 (2005).

28. Schiros, T. et al. Structure and bonding of the water-hydroxyl mixed phase on Pt(111). J. Phys. Chem. C. 111, 15003-15012 (2007). 
29. Ruan, C. Y., Lobastov, V. A., Vigliotti, F., Chen, S. Y. \& Zewail, A. H. Ultrafast electron crystallography of interfacial water. Science 304, 80-84 (2004).

30. Schiros, T. et al. Cooperativity in surface bonding and hydrogen bonding of water and hydroxyl at metal surfaces. J. Phys. Chem. C. 114, 10240-10248 (2010).

31. Du, Y. et al. Two pathways for water interaction with oxygen adatoms on $\mathrm{TiO}_{2}$ (110). Phys. Rev. Lett. 102, 096102 (2009).

32. Yamamoto, S. et al. In situ X-ray photoelectron spectroscopy studies of water on metals and oxides at ambient conditions. J. Phys. 20, 184025 (2008).

33. Lu, K. et al. High-efficiency PbS quantum-dot solar cells with greatly simplified fabrication processing via "solvent-curing". Adv. Mater. 30, e1707572 (2018).

34. Sizov, F. \& Plyatsko, S. Homogeneity range and nonstoichiometric defects in IV-VI narrow-gap semiconductors. J. Cryst. Growth 92, 571-580 (1988).

35. Kim, D., Kim, D. H., Lee, J. H. \& Grossman, J. C. Impact of stoichiometry on the electronic structure of PbS quantum dots. Phys. Rev. Lett. 110, 196802 (2013).

36. Hwang, G. W. et al. Identifying and eliminating emissive sub-bandgap states in thin films of PbS nanocrystals. Adv. Mater. 27, 4481-4486 (2015).

37. Chen, B.-A. et al. In situ identification of photo- and moisture-dependent phase evolution of perovskite solar cells. ACS Energy Lett. 2, 342-348 (2017).

38. Shuler, K. \& Laidler, K. The kinetics of heterogeneous atom and radical reactions. II. The recombination of hydroxyl radicals. J. Chem. Phys. 17, 1356-1357 (1949).

39. Huang, W., Manser, J. S., Kamat, P. V. \& Ptasinska, S. Evolution of chemical composition, morphology, and photovoltaic efficiency of $\mathrm{CH}_{3} \mathrm{NH}_{3} \mathrm{PbI}_{3}$ perovskite under ambient conditions. Chem. Mater. 28, 303-311 (2015).

40. Arai, $\mathrm{H}$. et al. Hydrogen bonding of water in 3-methylpyridine studied by $\mathrm{O} 1 \mathrm{~s}$ $\mathrm{X}$-ray emission and absorption spectroscopy. Phys. Chem. Chem. Phys. 14, 1576-1580 (2012).

41. Velasco-Velez, J. J. et al. The structure of interfacial water on gold electrodes studied by X-ray absorption spectroscopy. Science 346, 831-834 (2014).

42. Ning, Z. et al. Air-stable n-type colloidal quantum dot solids. Nat. Mater. 13, 822-828 (2014).

43. Van Huis, M. A. et al. Low-temperature nanocrystal unification through rotations and relaxations probed by in situ transmission electron microscopy. Nano Lett. 8, 3959-3963 (2008).

44. Evers, W. H. et al. Low-dimensional semiconductor superlattices formed by geometric control over nanocrystal attachment. Nano Lett. 13, 2317-2323 (2013).

45. Whitham, K. et al. Charge transport and localization in atomically coherent quantum dot solids. Nat. Mater. 15, 557-563 (2016)

46. Kramer, I. J. et al. Efficient spray-coated colloidal quantum dot solar cells. Adv. Mater. 27, 116-121 (2015).

47. Liu, Z. et al. High-efficiency hybrid solar cells based on polymer/ $\mathrm{PbS}_{\mathrm{x}} \mathrm{Se}_{1-\mathrm{x}}$ nanocrystals benefiting from vertical phase segregation. Adv. Mater. 25 5772-5778 (2013).

48. Shi, G. Z. et al. Finely interpenetrating bulk heterojunction structure for lead sulfide colloidal quantum dot solar cells by convective assembly. ACS Energy Lett. 4, 960-967 (2019).

49. Weidman, M. C., Smilgies, D. M. \& Tisdale, W. A. Kinetics of the selfassembly of nanocrystal superlattices measured by real-time in situ X-ray scattering. Nat. Mater. 15, 775-781 (2016).

50. Whitham, K., Smilgies, D.-M. \& Hanrath, T. Entropic, enthalpic, and kinetic aspects of interfacial nanocrystal superlattice assembly and attachment. Chem. Mater. 30, 54-63 (2017).

51. Yang, Z. Y. et al. Mixed-quantum-dot solar cells. Nat. Commun. 8, 1-9 (2017).

52. Proppe, A. H. et al. Picosecond charge transfer and long carrier diffusion lengths in colloidal quantum dot solids. Nano Lett. 18, 7052-7059 (2018)

53. Zhang, Y. et al. Photoexcited carrier dynamics in colloidal quantum dot solar cells: Insights into individual quantum dots, quantum dot solid films and devices. Chem. Soc. Rev. 49, 49-84 (2020).

54. Liu, Y., Kim, D., Morris, O. P., Zhitomirsky, D. \& Grossman, J. C. Origins of the stokes shift in PbS quantum dots: Impact of polydispersity, ligands, and defects. ACS Nano. 12, 2838-2845 (2018).

55. Voznyy, O. et al. Origins of stokes shift in PbS nanocrystals. Nano Lett. 17, 7191-7195 (2017).

56. Jones, M., Lo, S. S. \& Scholes, G. D. Quantitative modeling of the role of surface traps in $\mathrm{CdSe} / \mathrm{CdS} / \mathrm{ZnS}$ nanocrystal photoluminescence decay dynamics. Proc. Natl Acad. Sci. USA 106, 3011-3016 (2009).

57. Chuang, C. H. et al. Open-circuit voltage deficit, radiative sub-bandgap states, and prospects in quantum dot solar cells. Nano Lett. 15, 3286-3294 (2015).

58. Lu, K. et al. Packing state management to realize dense and semiconducting lead sulfide nanocrystals film via a single-step deposition. Cell Rep. Phys. Sci. 1, 100183 (2020).

59. Gilmore, R. H. et al. Epitaxial dimers and auger-assisted detrapping in $\mathrm{PbS}$ quantum dot solids. Matter 1, 250-265 (2019).
60. Hughes, B. K. et al. Synthesis and spectroscopy of PbSe fused quantum-dot dimers. J. Am. Chem. Soc. 136, 4670-4679 (2014).

61. Cui, J. et al. Colloidal quantum dot molecules manifesting quantum coupling at room temperature. Nat. Commun. 10, 5401 (2019).

62. Kwon, H. C. et al. Investigating recombination and charge carrier dynamics in a one-dimensional nanopillared perovskite absorber. ACS Nano. 12, 4233-4245 (2018).

63. Brown, P. R. et al. Energy level modification in lead sulfide quantum dot thin films through ligand exchange. ACS Nano. 8, 5863-5872 (2014).

64. Chuang, C. H., Brown, P. R., Bulovic, V. \& Bawendi, M. G. Improved performance and stability in quantum dot solar cells through band alignment engineering. Nat. Mater. 13, 796-801 (2014).

65. Bi, E. B. et al. Diffusion engineering of ions and charge carriers for stable efficient perovskite solar cells. Nat. Commun. 8, 15330 (2017).

66. Aqoma, H. et al. High-efficiency photovoltaic devices using trap-controlled quantum-dot ink prepared via phase-transfer exchange. Adv. Mater. 29, 1605756 (2017).

67. Azmi, R. et al. Highly efficient air-stable colloidal quantum dot solar cells by improved surface trap passivation. Nano Energy 39, 86-94 (2017).

68. Pradhan, S. et al. Trap-state suppression and improved charge transport in $\mathrm{PbS}$ quantum dot solar cells with synergistic mixed-ligand treatments. Small 13, 1700598 (2017).

69. Kresse, G. \& Furthmüller, J. Efficiency of ab-initio total energy calculations for metals and semiconductors using a plane-wave basis set. Comput. Mater. Sci. 6, 15-50 (1996)

70. Le Berre, M., Chen, Y. \& Baigl, D. From convective assembly to landau-levich deposition of multilayered phospholipid films of controlled thickness. Langmuir 25, 2554-2557 (2009).

71. Zhang, Y. et al. Hybrid quantum dot/organic heterojunction: a route to improve open-circuit voltage in PbS colloidal quantum dot solar cells. ACS Energy Lett. 5, 2335-2342 (2020).

\section{Acknowledgements}

This work was supported by the National Key Research Projects (Grant No. 2016YFA0202402), the National Natural Science Foundation of China (Grant Nos. 22161142003, 61911530158, 52002260, and 51803144), the Natural Science Foundation of Jiangsu Province of China (BK20200872). This work is supported by the Collaborative Innovation Center of Suzhou Nano Science and Technology, the 111 Project. The authors would like to thank SSRF (beamline 02B02) and TLS (beamline 20A1) for the allocation of synchrotron beamtime. This research also made use of resources at the Advanced Photon Source and the Center for Nanoscale Materials at Argonne National Laboratory, which was supported by the U.S. Department of Energy, Office of Science, Office of Basic Energy Sciences, under Contract No. DE-AC02-06CH11357. T.L. thanks the NIU start-up fund for support. H.W. and T.K. thank the Japan Society for the Promotion of Science (JSPS) for a Grant-in-Aid for Scientific Research (No. 16H03824 and 19H02534). Q.S. would like to thank the support of the MEXT KAKENHI (Grants 17H02736 and 20H02565). T.S. would like to thank the support of the MEXT KAKENHI (Grants 17H03536).

\section{Author contributions}

W.M. and Z.L. conceived the idea and supervised the project. G.S. designed the experiments. G.S. and H.W. performed the experiments and data analysis. Y.H.Z. and Q.S. conduct the TAS measurement. T.Z., B.C. X.Z., X.F.L., and S.D. contributed to the XPS measurement. C.C. and L.Z. measured the XAS spectra. R.J., X.N.M., and L.W. performed the DFT calculations. S.X. performed the MD simulations. X.Y.L. and T.L. conducted GISAXS measurements. Y.L. helped with the TEM measurements. T.S. helped with the FIB-TEM measurements. Y.N.Z., X.M., and Y.C. contributed to the SPLE and DS-PbS-I inks and devices. T.S., T.K., and H.S. construct the ideas and experimental design. G.S., W.M., and Z.L. co-wrote the manuscript.

\section{Competing interests}

The authors declare no competing interests.

\section{Additional information}

Supplementary information The online version contains supplementary material available at https://doi.org/10.1038/s41467-021-24614-7.

Correspondence and requests for materials should be addressed to Z.L. or W.M.

Peer review information Nature Communications thanks the anonymous reviewer(s) for their contribution to the peer review of this work.

Reprints and permission information is available at http://www.nature.com/reprints

Publisher's note Springer Nature remains neutral with regard to jurisdictional claims in published maps and institutional affiliations. 
(c) (i) Open Access This article is licensed under a Creative Commons Attribution 4.0 International License, which permits use, sharing, adaptation, distribution and reproduction in any medium or format, as long as you give appropriate credit to the original author(s) and the source, provide a link to the Creative Commons license, and indicate if changes were made. The images or other third party material in this article are included in the article's Creative Commons license, unless indicated otherwise in a credit line to the material. If material is not included in the article's Creative Commons license and your intended use is not permitted by statutory regulation or exceeds the permitted use, you will need to obtain permission directly from the copyright holder. To view a copy of this license, visit http://creativecommons.org/ licenses/by/4.0/.

(C) The Author(s) 2021 